\title{
Cirurgia para controle de danos: estado atual
}

\section{Damage control surgery: an update}

\author{
Rodrigo Camargo Leão Edelmuth'; Y Yuri dos Santos Buscariolli2; Marcelo Augusto Fontenelle Ribeiro Junior, TCBC-SP3
}

\author{
RE S U M O
}

\begin{abstract}
A cirurgia de controle de danos é um conceito amplamente aceito atualmente entre os especialistas em trauma abdominal quando se trata de doentes gravemente traumatizados. Nestes pacientes a morte decorre, na maioria das vezes, da instalação da tríade letal (hipotermia, coagulopatia e acidose) e não da incapacidade de reparar as graves lesões presentes. Nesta revisão, os autores abordam a tríade letal, em suas três fases, e enfatizam as medidas adotadas para preveni-las. Além de discutirem a indicação e o emprego da cirurgia para o controle de danos em seus vários estágios. A restauração dos padrões fisiológicos do doente na UTI, para que o mesmo possa ser submetido à operação definitiva e ao fechamento da cavidade abdominal, outro desafio no paciente traumatizado grave, também é discutida.
\end{abstract}

Descritores: Pacientes. Ferimentos e lesões. Terapêutica. Procedimentos cirúrgicos operatórios. Controle.

\section{INTRODUÇÃO}

O termo "controle de danos" tem origem na marinha norte-americana e se refere à capacidade dos navios militares de absorverem impacto e manterem sua integridade ao longo da missão. A utilização desse termo na medicina é voltada para a estratégia cirúrgica na qual reduz-se o tempo de cirurgia e sacrifica-se o reparo imediato de todas as lesões a fim de restaurar os parâmetros fisiológicos e não anatômicos no paciente instável. As técnicas utilizadas visam, prioritariamente, a controlar hemorragias, infecções e/ou vazamentos intestinais, biliares ou vesicais. Esse método consiste, portanto, em controlar de maneira não definitiva as lesões do doente e também é conhecido como laparotomia abreviada ${ }^{1-3}$.

Inicialmente essa abordagem foi utilizada em pacientes moribundos e vítimas de trauma, em especial nos traumas abdominais. Entretanto, esse método já é utilizado atualmente no tratamento de lesões torácicas, ortopédicas e vasculares de extremidades. Além disso, vem ganhando espaço em cenários não traumáticos, no qual o doente se encontra com acentuada instabilidade clínica e parâmetros fisiológicos bastante alterados, a ponto de impedir que a operação seja realizada até o $\mathrm{fim}^{3-7}$.

A ideia de controlar os danos de maneira temporária tem início no começo do Século 20, quando Pringle propôs a utilização de compressas na cavidade abdominal para tamponamento de hemorragias hepáticas. Em 1913, Halsted utilizou faixas de borracha juntamente com compressas, e essa técnica foi utilizada durante a Segunda Guerra Mundial.

No Brasil, há relatos do uso de "mechas de gaze asséptica" para hemostasia de lesão hepática grave em $1906^{5}$. Entretanto, foi somente no final do século que essa abordagem ganhou força. Em 1983, Stone et al. ${ }^{8}$ demonstraram que a taxa de mortalidade em pacientes exsanguinantes com coagulopatias caíra de $98 \%$ para 35\% com o emprego da laparotomia abreviada. Isso foi observado com o seguinte modelo de abordagem: laparotomia inicial com colocação de compressas intra-abdominais para conter hemorragia, ligadura de vasos e ressecção intestinal rápida sem anastomose ou formação de estomas. A operação era prontamente interrompida, uma vez que o paciente apresentasse coagulopatia. O reparo cirúrgico definitivo era realizado em um segundo momento, com o paciente estável e com sua coagulopatia corrigida. Dez anos após, em 1993, Rotondo et al. ${ }^{9}$ introduziram o termo "controle de danos".

\section{MÉTODOS}

Foi realizada uma revisão da literatura no do banco de dados da MEDLINE. Foram encontrados 179 artigos com os seguintes limites de busca no PUBMED: termo

\footnotetext{
1. Médico pesquisador do serviço de Cirurgia Geral do Hospital do Servidor Público Estadual - IAMSPE; 2. Médico pesquisador do grupo de Transplante e Cirurgia do Fígado do Hospital das Clínicas da Faculdade de Medicina da Universidade de São Paulo - HC-FMUSP; 3. Professor Titular, chefe da Disciplina de Cirurgia Geral e do Núcleo de Clínica Cirúrgica e Coordenador do Curso de Medicina da Universidade de Santo Amaro - UNISA.
} 
damage control no título, artigos publicados nos últimos dez anos, em inglês ou português, e somente em humanos. Além disso, buscamos nas referências dos artigos selecionados, outros trabalhos relevantes para essa revisão, assim como sugestão de especialistas na área. Através de nossos critérios de inclusão selecionamos um total de 42 artigos, que foram utilizados como base para a realização desta revisão.

\section{Tríade letal}

Burch et al. ${ }^{10}$ descreveram esse ciclo vicioso no qual há um desarranjo metabólico importante que leva à exaustão fisiológica. A taxa de hemorragia secundária, a coagulopatia, cresce para $40 \%$ quando associada à hipotensão, para $50 \%$ quando há hipotermia e para $60 \%$ na presença de acidose. Podendo chegar à exorbitante taxa de $98 \%$ na presença de toda a tríade letal ${ }^{11}$.

\section{Hipotermia}

É comum nos pacientes vítimas de trauma, em conseqüência de acentuada perda sanguínea, exposição do doente, reanimação com fluidos não aquecidos ou perda da capacidade de termorregulação em pacientes intoxicados ou com danos neurológicos. Esses fatos acarretam a hipoperfusão tecidual resultante da vasoconstrição periférica, que tem início a partir de uma intensa descarga simpática. A redução na oferta de oxigênio e a conversão do metabolismo aeróbico em anaeróbico culminam em acidose metabólica.

A hipotermia torna-se, clinicamente, importante quando a temperatura corpórea está abaixo de $36^{\circ} \mathrm{C}$ por mais de quatro horas, e uma temperatura abaixo de $35^{\circ} \mathrm{C}$ está relacionada com um pior prognóstico. Há relatos evidenciando mortalidade de $100 \%$ em pacientes com temperatura abaixo de $32^{\circ} \mathrm{C}^{1,2,12}$. A hipotermia pode causar arritmias ventriculares, diminuição da pós-carga, maior resistência vascular periférica e desvio da curva de dissociação de oxigênio para a esquerda. Há também correlações de hipotermia com supressão do sistema imune.

\section{Acidose metabólica}

A acidose é um preditor importante da gravidade de lesão abdominal e de prognóstico do paciente. Um $\mathrm{pH}$ baixo por tempo prolongado é sinal de prognóstico ruim e $\mathrm{pH}<7,2$ está relacionado com alta mortalidade ${ }^{1}$.

A acidose metabólica agrava o quadro de coagulopatia pela inativação de fatores de coagulação $\mathrm{pH}$ dependentes ${ }^{2}$. Além disso, a acidose pode sobrecarregar o sistema respiratório na tentativa de realizar uma alcalose respiratória compensatória. Essa alteração também pode levar à diminuição da contratilidade miocárdica e reduzir a pós-carga.

Múltiplas transfusões sanguíneas, clampeamento da aorta e função miocárdica reduzida também contribuem para a piora da acidose.

\section{Coagulopatia}

A maioria dos pacientes politraumatizados encontra-se em um estado de hipercoagulabilidade ou com parâmetros de coagulação pouco alterados. Entretanto, os $10 \%$ restantes, são doentes com hipocoagulabilidade e com pior prognóstico.

Anormalidades de coagulação após o trauma são fatores independentes de alta mortalidade. Um tempo de protrombina (TP) anormal inicial aumenta o risco de morte em 35\%, enquanto um tempo anormal de tromboplastina parcial ativada (TTPa) aumenta esse risco em 326\%1,13. Moore et al. ${ }^{14}$ descreveram que coagulopatia grave (TP > duas vezes seu valor normal e TTPa $>$ duas vezes seu valor normal) é um importante preditor de morte.

A hipotermia é um importante fator no desenvolvimento do distúrbio de coagulação, porque: inibe a interação do fator de von Willebrand com as glicoproteínas plaquetárias, ocasionando disfunção plaquetária; inativa os fatores de coagulação temperatura-dependentes; induz alterações no sistema fibrinolítico e gera anormalidades endoteliais. Em temperaturas menores que $35^{\circ} \mathrm{C}$, os fatores de coagulação entram em um estado de hipometabolismo agravando o quadro clínico ${ }^{1,2}$.

A administração excessiva de fluidos, na tentativa de reposição volêmica no paciente instável, é uma importante causa de coagulopatia devido à hemodiluição que decorre tanto da infusão acentuada de cristaloides quanto das transfusões sanguíneas ${ }^{1,2,15}$.

A hemodiluição e a hipotermia são os fatores evitáveis mais frequentes e, portanto, o médico deve estar sempre atento em manter o paciente aquecido com mantas térmicas, fluídos aquecidos e evitar a desnecessária infusão fluídos'

Estágios da cirurgia para controle de danos

A cirurgia de controle de danos pode ser dividida em cinco estágios: seleção do paciente, operação abreviada, correção dos parâmetros fisiológicos na UTI, reoperação programada e fechamento da parede abdominal. Entretanto alguns autores, como Parreira ${ }^{5}$ e Rotondo ${ }^{16}$, não consideram a seleção de paciente e o fechamento da parede abdominal como estágios isolados e, portanto, dividem a cirurgia de controle de danos em três momentos.

\section{Seleção do paciente}

Não há consenso absoluto quanto aos critérios que devem ser utilizados para a realização da cirurgia de controle de danos. Entretanto, não há dúvida de que essa escolha deve ser tomada precocemente. Além disso, é imprescindível que haja disponibilidade de vagas em UTI, equipe técnica qualificada e centros cirúrgicos especializados, ou que pelo menos tenham supervisão cirúrgica contínua ${ }^{5}$. O cirurgião deve ficar atento aos sinais e sintomas do paciente e evitar alterações fisiológicas limítrofes, pois essas podem representar a perda do momento ideal para a realização da cirurgia de controle de 
danos. A instabilidade hemodinâmica manifestada pela hipotensão, taquicardia, taquipneia e alteração do estado de consciência devem alertar o médico para a potencial necessidade de realizar a laparotomia abreviada. Doentes que apresentem coagulopatia e/ou hipotermia são prováveis candidatos a essa abordagem. Alguns autores também propõem que essa decisão seja baseada na magnitude das lesões e no mecanismo do trauma. Vitimas de lesões vasculares abdominais importantes, lesões múltiplas de órgãos e com hemorragias multifocais em diferentes cavidades também devem ser considerados para esse tipo de abordagem ${ }^{16-18}$

Em 2002, Parreira et al. ${ }^{19}$ descreveram um sistema de indicação baseado na probabilidade de hemorragia letal e se utilizam dessas informações para interromper a operação no doente grave, fornecendo assim dados objetivos para a indicação da laparotomia abreviada (Tabela 1).

Kairinos et al. ${ }^{12}$ propuseram critérios matemáticos que visam a estimar a mortalidade do doente e, assim, auxiliar o raciocínio clínico do cirurgião. A equação proposta, $X=[(0,012)$.idade $]-[(0,707)$. $\mathrm{pH}]-[(0,032)$ temperatura] $+6,002$, demonstrou uma mortalidade inevitável naqueles pacientes com $X>0,5$ (sensibilidade de $25 \%$ e valor preditivo positivo de $100 \%)$. As três variáveis dessa fórmula são os principais fatores pré-operatórios preditivos de mortalidade, sendo a idade o principal deles. Esse mesmo grupo demonstrou uma mortalidade de $100 \%$ em pacientes submetidos a laparotomia abreviada com mais de 58 anos de idade.

Além dessas variáveis, Asensio et al. ${ }^{18}$ demonstraram por meio de um estudo retrospectivo que alguns sinais clínicos também predizem mortalidade em pacientes com hemorragia importante: pupilas não reagentes, ausência de ventilação espontânea, pulso carotídeo não palpável, ausência de movimento de extremidades e ausência de ritmo sinusal.
Sabe-se que a tomada de decisão no momento adequado e para os pacientes que têm indicação não só é fundamental, como também decisiva para o sucesso dessa abordagem. Um estudo retrospectivo com 532 pacientes demonstrou uma correlação direta entre a diminuição de laparotomias abreviadas em um período de dois anos, de $36,3 \%$ para $8,8 \%$ e uma queda significativa na mortalidade e redução da utilização de recursos e custos hospitalares. Esse resultado corrobora a ideia da necessidade de critérios rígidos para a indicação da operação ${ }^{20}$.

Em suma, não há critérios bem estabelecidos para indicação da cirurgia de controle de danos e, portanto, o cirurgião deve avaliar o cenário completo, tendo em mente todos os dados fisiológicos do doente, a gravidades de suas lesões e o mecanismo de trauma. Pelas experiências ruins, muitos cirurgiões experientes aprenderam que é melhor recuar do que presenciar o paciente atingir seu limite fisiológico ${ }^{17}$. Muitas vezes essa decisão é tomada antes mesmo de o paciente entrar no centro cirúrgico. Os principais critérios podem ser vistos na tabela 21,15,16,19, 21-25.

\section{Operação abreviada}

Esse procedimento tem por objetivo controlar rapidamente os focos de hemorragia e infecção e diminuir as contaminações no menor tempo possível.

\section{Controle da hemorragia}

Após uma laparotomia mediana xifopúbica a hemostasia deve ser realizada, e pode ser feita com a colocação de compressas nos quatro quadrantes, ligadura ou clampeamento de vasos e/ou utilização de balões para tamponamento. As compressas podem ser trocadas durante a operação e, eventualmente, podem ser deixadas na cavidade abdominal e retiradas somente na reoperação programada. Os reparos vasculares complexos não devem ser realizados nesse momento ${ }^{1-3}$.

Tabela 1 - Probabilidade de hemorragia letal, em \%, calculada com base na pressão arterial sistólica aferida no início da operação e o volume de concentrado de hemácias infundidas durante a operação (Parreira et al. 2002).

\begin{tabular}{lrrrrrrrrrrrr}
\hline CH / PA & 900 & 1200 & 1500 & 1800 & 2100 & 2400 & 2700 & 3000 & 3300 & 3600 & 3900 & $\mathbf{4 2 0 0}$ \\
\hline 0 & 67 & 72 & 77 & 81 & 84 & 87 & 90 & 92 & 93 & 95 & 96 & 97 \\
10 & 58 & 64 & 69 & 74 & 78 & 82 & 85 & 88 & 90 & 92 & 94 & 95 \\
20 & 48 & 54 & 60 & 66 & 71 & 75 & 80 & 83 & 86 & 89 & 91 & 93 \\
30 & 38 & 44 & 50 & 56 & 62 & 67 & 72 & 77 & 81 & 84 & 87 & 90 \\
40 & 29 & 35 & 40 & 46 & 52 & 58 & 64 & 69 & 74 & 78 & 82 & 85 \\
50 & 22 & 26 & 31 & 36 & 42 & 48 & 54 & 60 & 66 & 71 & 75 & 80 \\
60 & 16 & 19 & 23 & 28 & 33 & 38 & 44 & 50 & 56 & 62 & 67 & 72 \\
70 & 11 & 14 & 17 & 21 & 25 & 29 & 35 & 40 & 46 & 52 & 58 & 64 \\
80 & 8 & 10 & 12 & 15 & 18 & 22 & 26 & 31 & 37 & 42 & 48 & 54 \\
90 & 5 & 7 & 8 & 10 & 13 & 16 & 19 & 23 & 28 & 33 & 38 & 44 \\
100 & 4 & 5 & 6 & 7 & 9 & 11 & 14 & 17 & 21 & 25 & 30 & 35 \\
110 & 2 & 3 & 4 & 5 & 6 & 8 & 10 & 12 & 15 & 18 & 22 & 26 \\
\hline
\end{tabular}

$\mathrm{CH}$ - volume de concentrado de hemácias transfundido, em mililitros.

PA - Pressão arterial sistólica aferida no início da operação, em $\mathrm{mmHg}$. 
Tabela 2 - Principais critérios para a seleção de pacientes.

\begin{tabular}{|c|c|c|c|c|c|c|c|c|c|c|}
\hline $\begin{array}{l}\text { Autor } \\
\text { Ano }\end{array}$ & $\begin{array}{c}\text { Garrison } \\
\text { et al. } .^{23} \\
1996\end{array}$ & $\begin{array}{c}\text { Cushmann } \\
\text { et al.22 } \\
1997\end{array}$ & $\begin{array}{c}\text { Cosgriff } \\
\text { et al. }{ }^{21} \\
1997\end{array}$ & $\begin{array}{c}\text { Rotondo } \\
\text { et al. }{ }^{16} \\
1997\end{array}$ & $\begin{array}{c}\text { Krishna } \\
\text { et al..24 } \\
1998\end{array}$ & $\begin{array}{l}\text { Asensio } \\
\text { et al. }{ }^{18} \\
2001\end{array}$ & $\begin{array}{l}\text { Parreira } \\
\text { et al. }{ }^{19} \\
2002\end{array}$ & $\begin{array}{l}\text { Stalhschmidt } \\
\text { et al.25 } \\
2006\end{array}$ & $\begin{array}{l}\text { Germanos } \\
\text { et al. }{ }^{1} \\
2008\end{array}$ & $\begin{array}{l}\text { Matsumoto } \\
\text { et al. } .^{15} \\
2010\end{array}$ \\
\hline $\begin{array}{l}\text { Tipo de estudo e } \\
\text { número de pacientes }\end{array}$ & $\begin{array}{l}\text { Prospectivo } \\
\quad(n=70)\end{array}$ & $\begin{array}{l}\text { Retrospectivo } \\
\quad(n=53)\end{array}$ & $\begin{array}{l}\text { Prospectivo } \\
\quad(n=58)\end{array}$ & Revisão & $\begin{array}{l}\text { Retrospectivo } \\
\quad(n=40)\end{array}$ & $\begin{array}{l}\text { Retrospectivo } \\
\quad(n=548)\end{array}$ & $\begin{array}{l}\text { Prospectivo } \\
\quad(n=74)\end{array}$ & Revisão & Revisão & $\begin{array}{l}\text { Retrospectivo } \\
\qquad(\mathrm{n}=34)\end{array}$ \\
\hline Base dos critérios & $\begin{array}{l}\text { Utilização de } \\
\text { compressas para m } \\
\text { hemostasia e } \\
\text { análise de diversas } \\
\text { variáveis entre } \\
\text { pacientes sobrevi- } \\
\text { ventes e não } \\
\text { sobreviventes }\end{array}$ & $\begin{array}{l}\text { Indicadores de } \\
\text { morte em vítima de } \\
\text { ferimentos pene- } \\
\text { trantes de vasos } \\
\text { ilíacos }\end{array}$ & $\begin{array}{c}\text { Avaliação de } \\
\text { efatores preditivos } \\
\text { de coagulopatia }\end{array}$ & - & $\begin{array}{c}\text { Paciente com } \\
\text { trauma de múlti- } \\
\text { plas vísceras.ISS > } \\
35 \text { eGlasgow }>9\end{array}$ & $\begin{array}{l}\text { Perda sanguínea } \\
\text { > } 2 \mathrm{~L} \text { durante a } \\
\text { cirurgia; Transfu- } \\
\text { são concentrado } \\
\text { de hemácias e" } \\
1,5 \mathrm{~L} \text { durante } \\
\text { ressuscitação ; } \\
\text { pctes com diag- } \\
\text { nóstico de } \\
\text { exsanguinação ** }\end{array}$ & $\begin{array}{l}\text { Vítimas de ferimentos } \\
\text { penetrantes de tronco } \\
\text { com PAS }<90 \mathrm{mmHg} \text {, } \\
\text { hemorragia e que } \\
\text { sobreviveram até o } \\
\text { tratamento defnitivo }\end{array}$ & s & - & $\begin{array}{l}\text { Pacientes com trau- } \\
\text { mas grave de bacia e } \\
\text { abdomen } \\
\text { hemodinamicamente } \\
\text { instáveis após } \\
\text { ressuscitação com } \\
\text { fluidos. }\end{array}$ \\
\hline Temperatura & - & $<35^{\circ} \mathrm{C}$ & $<34^{\circ} \mathrm{C}$ & $<35^{\circ} \mathrm{C}$ & $<33^{\circ} \mathrm{C}$ & $<34^{\circ} \mathrm{C}$ & - & $<34^{\circ} \mathrm{C}$ & $<34^{\circ} \mathrm{C}$ & $<35,5^{\circ} \mathrm{C}$ \\
\hline Base excess & - & $<-6 \mathrm{mEg} / \mathrm{L}$ & 一 & - & $<-12 \mathrm{mEq} / \mathrm{L}$ & - & $<-10 \mathrm{mEq} / \mathrm{L}$ & & - & $<-7,5 \mathrm{mmol} / \mathrm{L}$ \\
\hline $\mathrm{pH}$ & $<7,2$ & $<7,3$ & $<7,1$ & $<7,3$ & $\begin{array}{l}\text { Acidose metabólica } \\
\text { grave }\end{array}$ & $<7,2$ & $<7,25$ & $<7,2$ & $<7,2$ & - \\
\hline Pressão arterial & $\begin{array}{l}\text { Hipotensão prolongada } \\
\qquad \text { (>70 min) }\end{array}$ & 一 & $\mathrm{PAS}<70 \mathrm{mmHg}$ & - & $<80(60-90) \mathrm{mmHg}$ & & $\begin{array}{c}\text { PAS }<110 \mathrm{mmHg} \text { no } \\
\text { início da cirurgia }\end{array}$ & & $\mathrm{PAS}<70 \mathrm{mmHg}$ & $\mathrm{PAS}<90 \mathrm{mmHg}$ \\
\hline Alterações sanguíneas & $\begin{array}{c}\mathbf{s} \quad 15 \text { bolsas } \\
\text { de } \mathrm{CH}\end{array}$ & 一 & Coagulopatia > & $\begin{array}{c}>10 \text { bolsas de } \mathrm{CH} \\
\text { (> } 4 \mathrm{~L} \text { de perda } \\
\text { sanguínea) } \\
\text { Coagulopatia }\end{array}$ & $\begin{array}{l}\text { H Perda sanguínea } \\
>4 \mathrm{~L}(2,5-6)^{\star} \\
\text { INR }>2,2 \\
(1,6-3,2)^{\star}\end{array}$ & $\begin{array}{l}\text { Perda sanguínea } \\
\text { estimada }>5 \mathrm{~L} \\
\text { ou }>15 \mathrm{~mL} / \mathrm{min} \\
\text { Reposiçãa volêmica } \\
\text { total }>12 \mathrm{~L} \\
\text { Transfusão }>4 \mathrm{~L} \mathrm{CH}\end{array}$ & $\begin{array}{l}\text { Choque persistente } \\
\text { Transfusão de } \\
\text { CH>1200 ml }\end{array}$ & $\begin{array}{l}\text { Incapacidade de } \\
\text { hemostasia devido } \\
\text { ao desenvolvimento } \\
\text { de coagulopatia e } \\
\text { inacessibilidade } \\
\text { aos vasos; } \\
\text { Coagulopatia }\end{array}$ & $\begin{array}{l}\text { Instabilidade } \\
\text { hemodinâmica } \\
\text { (Taquicardia, } \\
\text { arritmias, pulsos } \\
\text { carotídeos fracos) } \\
>10 \text { bolsas } \\
\text { de CH }\end{array}$ & - \\
\hline TTPa & $>60 \mathrm{~s}$ & 一 & $\begin{array}{l}\text { Duas vezes maior } \\
\text { seu valor padrão }\end{array}$ & - & - & - & - & - & $>19 s$ & - \\
\hline TP & $>19 s$ & 一 & $\begin{array}{l}\text { Duas vezes maior } \\
\text { seu valor padrão }\end{array}$ & 一 & 一 & - & - & - & $>19 s$ & - \\
\hline ISS & $>35$ & & $>25$ & - & $>51(41-61)^{*}$ & $<20 * * *$ & - & $>35$ & - & - \\
\hline Outros & - & - & - & $\begin{array}{l}\text { Tempo de } \\
\text { operação } \\
>90 \text { min }\end{array}$ & $\begin{array}{c}\text { Combinação de } \\
\mathrm{T}=33,5^{\circ} \mathrm{C} \\
-35,5^{\circ} \mathrm{C} \text { e } \\
\mathrm{BE}<-5-12 \mathrm{mEq} / \mathrm{L}\end{array}$ & $\begin{array}{l}\text { Ventilação espontânea; } \\
\text { Transfusão de CH > 4L; } \\
\text { ausência de } \\
\text { L toracotomia de } \\
\text { emergência; ausência } \\
\text { de lesão vascular } \\
\text { abdominal*** }\end{array}$ & ; & $\begin{array}{c}\text { Choque } \\
\text { hipovolêmico } \\
\text { por mais que } \\
70 \text { min }\end{array}$ & $\begin{array}{l}\text { Tempo de operação } \\
>90 \mathrm{~min}\end{array}$ & $\begin{array}{l}\text { O cirurgião pode } \\
\text { optar pela } \\
\text { CCD com a } \\
\text { presença de } \\
1 \text { ou } 2 \text { dos critérios }\end{array}$ \\
\hline
\end{tabular}

$* p>0,005$

**perda sanguínea inicial $>40 \%$ e hemorragia ativa que, se não controlada cirurgicamente, será a causa de óbito do paciente

***fatores de risco independentes diretamente relacionados a sobrevivência do doentes

TP: tempo de protrombina; TTPa: tempo de trombloplastina parcial ativada; CCD: cirurgia de controle de danos; ISS: "injury severity score"; PAS: pressão arterial sistólica; CH: concentrado de hemácias 

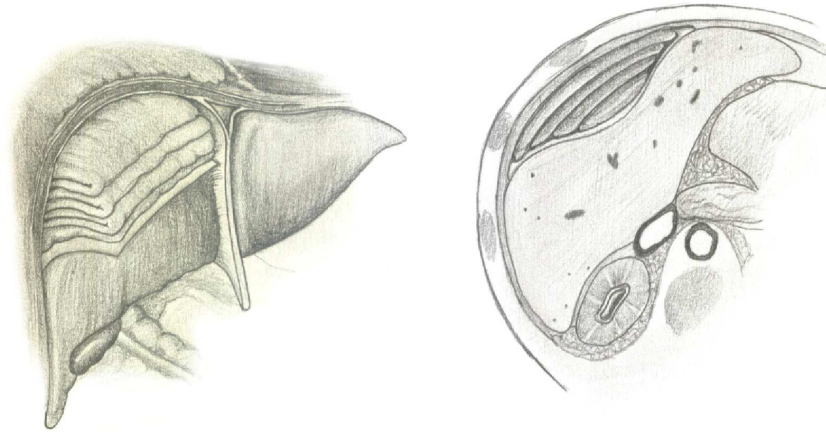

Figura 1 - Colocação de compressas para tamponamento hepático.

Os sangramentos hepáticos podem ser controlados por meio da digitoclasia, ligadura direta dos vasos e/ou tamponamento com compressas. Essas devem ser postas de maneira a criar vetores de força e não devem ser "empurradas" desordenadamente (Figura 1) 17,26-28. Além disso, são ineficazes para hemorragias provenientes de grandes vasos. Nesses casos a sutura do parênquima deve ser realizada. Os balões hepáticos, confeccionados a partir de sondas nasogástricas, drenos de Penrose, balão de Sengstaken Blakemore ou até mesmo a sonda de Foley, podem ser insuflados dentro do parênquima hepático em casos de hemorragias mais vultuosas, que não cessam com a digitoclasia.

Ressecções regradas e mais complexas devem ser evitadas, e os sangramentos esplênicos e/ou renais também podem ser tratados com sutura do parênquima. Se houver necessidade de um procedimento mais complexo, a ressecção do órgão está indicada ${ }^{29}$.

Através de manobras de rotação visceral são avaliadas as estruturas retroperitoneais à procura de possíveis hematomas perirrenais, retro-hepáticos e pélvicos, pois estes não devem ser explorados, e sim tamponados e encaminhados para embolização angiográfica ${ }^{15}$.

A hemorragia causada pelas lesões de grandes vasos possui muitas variáveis que devem ser observadas para que o tratamento adequado ao paciente grave possa ser feito: as lesões passíveis de correção com sutura simples devem ser prontamente tratadas; nas lesões complexas, um shunt ou uma ligadura estão indicados. Vale ressaltar que a ligadura da aorta, da artéria mesentérica superior e da ilíaca externa têm graves consequências e, portanto, estão contraindicadas. Com exceção da veia cava inferior cranial às artérias renais, todas as outras veias da cavidade abdominal podem ser ligadas 27,28.

Um estudo multicêntrico, randomizado e controlado, (CRASH-II), demonstrou resultados satisfatórios relacionados ao ácido tranexâmico, um agente antifibrinolítico. O uso precoce (d" uma hora pós trauma) reduziu a taxa de mortalidade por exsanguinação (5,3\% vs. 7,7\%; RR 0,68, 95\% IC: 0,57-0,82; $p<0,0001)$, assim como, o uso entre a primeira e a terceira hora pós trauma $(4,8 \%$ vs. $6,1 \%$; RR 0,79, IC 0,64-0,97; $p=0.03)$. Entretan- to, vale ressaltar que os autores contraindicam o uso após três horas, devido a uma maior mortalidade secundária ao sangramento (4,4\% vs. 3,1\%; RR 1,44, IC 1,12-1,84; $\mathrm{p}=0.004)^{30}$. Autores, como Murthi et al., questionam esse ensaio clínico como sendo controverso. Apesar disso, dados provenientes da experiência do exército britânico corroboram para o uso do ácido tranexâmico ${ }^{11}$.

\section{Controle de contaminação}

A segunda meta é controlar os possíveis focos de contaminação. O cirurgião deve inspecionar toda a extensão das alças intestinais, do ângulo de Treitz ao reto. A correção das lesões deve ser feita com suturas simples, se possível. Quando as lesões forem mais extensas, a ressecção do segmento intestinal traumatizado pode ser feita, e os cotos, distal e proximal, ligados. Pinças não traumáticas para alças ou fitas cardíacas também podem ser utilizadas para ocluir as alças. Quando há múltiplas lesões em um seguimento menor que $50 \%$ de todo o comprimento do intestino uma única ressecção pode ser realizada. As anastomoses ou estomas não devem ser feitos nesse instante ${ }^{1,2,5}$. As lesões ureterais também podem ser tratadas com shunts temporários ${ }^{1}$. Antes do término da operação, deve-se irrigar as cavidades abdominal e pélvica com solução salina aquecida.

Como existe indicação absoluta de uma segunda operação, o fechamento da parede abdominal deve ser feito de maneira temporária. Para isso diferentes técnicas estão disponíveis: síntese com pinças de Backhaus, "Bolsa de Bogotá", campos plásticos estéreis adesivos ou curativos com aspiração por vácuo contínuo (Figura 2).

\section{Recuperação na UTI}

O objetivo dessa etapa é restaurar os parâmetros fisiológicos do paciente dentro da unidade de terapia intensiva. A reposição volêmica, a correção da acidose, da hipotermia e da coagulopatia são essenciais para a reani-

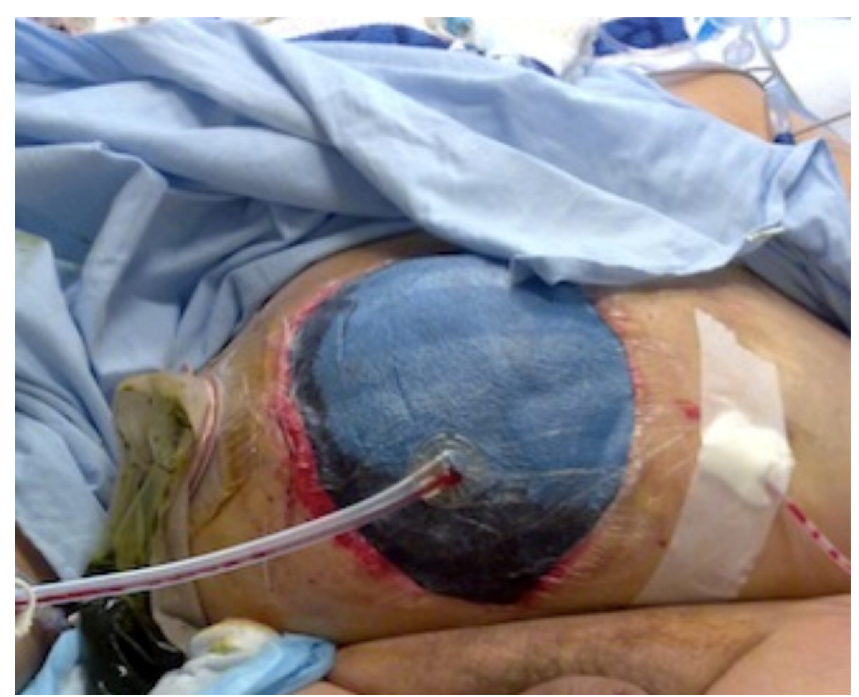

Figura 2 - Colocação do curativo à vácuo. 
mação do doente grave para que possa ser submetido a nova operação. Esse período pode levar de 24 a 48 horas. Entretanto, não existe consenso absoluto quanto aos parâmetros a serem atingidos. A tabela 3 mostra alguns valores que podem servir como metas a serem atingidas.

\section{Correção da acidose}

A acidose desses pacientes resulta, na maioria dos casos, da má perfusão tecidual sistêmica que interfere diretamente no metabolismo aeróbico. Por sua vez, a hipóxia é resultado de dois importantes fatores: a hipotermia e a acentuada perda sanguínea. Portanto, a acidose irá se autocorrigir a partir do momento que a oferta de oxigênio for suficiente para a demanda e a temperatura adequada for atingida ${ }^{2,5}$. O médico deve visar melhorar parâmetros como concentração de hemoglobina, saturação de $\mathrm{O}_{2}$, pressão parcial de $\mathrm{O}_{2}$ e débito cardíaco. Isso deve ser feito mediante uma reposição volêmica adequada juntamente com o controle da hipotermia. O uso de bicarbonato nesses casos passa, então, para o segundo plano. Estudos preliminares têm mostrado resultados interessantes com o uso tris(hidroximetil)aminometano, um aminoálcool capaz de captar íons de hidrogênio e corrigir, em teoria, a acidose 5 $^{53}$.

O quadro clínico do paciente é muito importante para avaliar as metas a serem atingidas. Entretanto, essa avaliação fica prejudicada no doente grave pela resposta neuroendócrina após o trauma, pois leva à retenção hídrica, oligúria e taquicardia.

\section{Hipotermia}

O médico deve sempre tentar prevenir a hipotermia, pois sua prevenção é mais fácil que sua correção. Para isso algumas medidas podem ser tomadas, como rápida finalização da laparotomia, retirada de roupas úmidas, diminuição da exposição do paciente, aque-

Tabela 3 - Metas a serem atingidas na UTI.

\begin{tabular}{lr}
\hline Pressão sistólica & $90 \mathrm{mmHg}$ \\
Lactato sérico & $<2,5 \mathrm{mmol} / \mathrm{l}$ \\
Base excess & $-4 \mathrm{mmol} / \mathrm{L}$ \\
Hemoglobina & $10 \mathrm{~g} / \mathrm{dL}$ \\
Hematócrito & $>30 \%$ \\
Temperatura & $>35^{\circ} \mathrm{C}$ \\
Tempo de protombina & $<15 \mathrm{~s}$ \\
TTPa* & $<35 \mathrm{~s}$ \\
Plaquetas & $50.000 / \mathrm{mm}^{3}$ \\
Fibrinogênio & $>100 \mathrm{mg} / \mathrm{dL}$ \\
Cálcio sérico & $>0,9 \mathrm{mmol} / \mathrm{L}$ \\
Índice cardíaco & $>3 \mathrm{~L} / \mathrm{min} / \mathrm{m}^{2}$ \\
Saturação O2 & $>95 \%$ \\
FIO2 " & $<50 \%$ \\
Dosagem de inotrópicos & Baixa
\end{tabular}

*Tempo de tromboplastina parcialmente ativada.

" Frações inspiradas de oxigênio. cimento do ambiente e de todos os fluídos antes da administração.

Uma vez instalada, a hipotermia deve ser prontamente corrigida, pois sua correção é um fator de extrema importância para o controle das demais alterações fisiológicas. Na tentativa de revertê-la, devem ser adotadas, além das medidas já descritas, o emprego de: colchões térmicos, sistemas de aquecimento pela circulação de ar aquecido, irrigação de sondas gástricas e vesicais com solução salina aquecida a $39-40^{\circ} \mathrm{C}$ e/ou irrigação das cavidades torácica ou abdominal através de drenos de tórax ou cateteres de diálise peritonial $2,4,28$.

Jaunoo et al. recomendam que a temperatura do paciente deve ser maior que $37^{\circ} \mathrm{C}$ após quatro horas de entrada na UTI ${ }^{2}$. Caso não haja resposta a estas medidas e a temperatura mantenha-se abaixo de $35^{\circ} \mathrm{C}$, deve-se pensar no uso de diversos drenos de tórax com solução aquecida. O aquecimento arteriovenoso contínuo pode ser utilizado em temperaturas menores que $33^{\circ} \mathrm{C}$.

Coagulopatia e reposição volêmica - "O conceito da Damage Control Ressuscitation e da Hipotensão Permissiva"

A correção da coagulopatia é um ponto central para um melhor prognóstico desses pacientes graves. Por sua vez, o ponto mais importante para a correção da coagulopatia é o tratamento da hipotermia.

O médico deve ter em mente que o uso de cristaloides nos pacientes em estado de hipocoagulabilidade é extremamente limitado, pois uso de solução salina a 0,9\% e de Ringer Lactato aumenta a lesão por reperfusão, induz adesão leucocitária e acarreta a hemodiluição ${ }^{13}$. A reposição de fatores de coagulação e plaquetas é, portanto, fundamental nesses cenários. Para a correção da coagulopatia podem ser utilizados plasma fresco, concentrado de plaquetas, fator recombinante Vlla, crioprecipitados, ácido tranexâmico e/ou reposição de cálcio ${ }^{30,31}$.

Diferentemente de estratégias convencionais de reposição volêmica, esses pacientes necessitam de volumosas transfusões, conhecidas como "protocolos de transfusão maciça" / "damage control ressuscitation". Alguns autores sugerem a administração na proporção 1:1:1 (concentrado de hemácias, plasma fresco e plaquetas) e a regra dos 10 (dez unidades de cada em 24 horas)2,13. Os protocolos de transfusão volumosa ganharam muita força na última década, após as experiências militares no Iraque e Afeganistão em 2004/200511. Um estudo retrospectivo demonstrou queda de $47 \%$ na mortalidade em vítimas de conflitos militares que receberam concentrado de hemácias e plasma fresco na proporção 1:1 quando comparado com aqueles cuja reposição foi feita na proporção de $1: 8^{31}$.

Há quem discorde desta conduta e recomende que o uso de plaquetas deva ser restringido à utilização de uma bolsa para cada quatro a seis concentrados de 
hemácias, argumentando que a proporção 1:1:1 é muito similar à administração de sangue total ${ }^{32}$. É importante também salientar que o uso de derivados de sangue pode ter consequências desastrosas, devido as suas propriedades inflamatórias e imunomodulatórias. A lesão pulmonar aguda (TRALI- transfusion related acute lung injury) é a principal e mais temida consequência ${ }^{33,34}$.

Além disso, todos os estudos recentes são retrospectivos e possuem vieses sobre a mortalidade. Cabe, portanto, ao cirurgião do trauma, uma avaliação criterioso sobre o tema, enquanto estudos prospectivos são realiza$\operatorname{dos}^{11}$.

No entanto, não restam dúvidas quanto à importância da ressuscitação no controle de danos (damage control resuscitation), tendo como meta a euvolemia ou até mesmo um certo grau de hipotensão, desde que associada à boa oxigenação periférica. O conceito de hipotensão permissiva pode e deve ser utilizado, evitando-se o uso de fluidos desnecessários e suas consequências, como a hemodiluição e hipotermia. O cirurgião do trauma deve ter em mente que o uso de derivados de sangue, associado à noção da hipotensão permissiva é, provavelmente um divisor de águas na cirurgia do trauma. No entanto, mais estudos ainda são necessários para concluir qual a melhor relação quantitativa entre concentrados de hemácias, plasmas frescos congelados e bolsas de plaquetas. Certamente, o antigo conceito de ressuscitação volêmica agressiva com altos volumes de cristaloide, visando a parâmetros suprafisiológicos, deve ser evitado ${ }^{35}$.

Devido ao alto custo do fator recombinante VIla, sua utilização ainda é questionada e estudos que avaliem seu custo-benefício ainda são necessários. Os autores favoráveis à administração do fator recombinante Vlla recomendam o seu emprego em conjunto com as primeiras bolsas de sangue e ao longo do tratamento conforme necessário ${ }^{13,32}$.

\section{Outros procedimentos}

Durante esse período é indicado a antibioticoterapia profilática, a sedação para melhorar o padrão respiratório e o controle da pressão intra-abdominal, trazendo mais conforto para o paciente. A fim de evitar a síndrome compartimental abdominal, deve-se aferir a pressão da cavidade através da sonda vesical ou gástri$\mathrm{ca}^{5}$. O diagnóstico é feito quando a pressão excede $20 \mathrm{mmHg}$ com evidências de disfunção orgânica ${ }^{3}$.

A arteriografia também pode ser considerada para tratar focos hemorrágicos, que foram temporariamente tamponados na operação inicial com compressas, nos casos de lesões hepáticas graves e/ou trauma de bacia ${ }^{36}$.

Outras medidas importantes de terapia intensiva mantêm uma relação direta com menores taxas de morbimortalidade e estão indicadas. A ventilação mecânica protetora com baixo volume corrente e um controle glicêmico não muito restrito $(<180 \mathrm{mg} / \mathrm{dL})$ são clássicos exemplos recentes disso ${ }^{37,38}$.

\section{Reoperação Programada}

Não há período mínimo ou máximo para o retorno do paciente ao centro cirúrgico. Alguns afirmam que isso ocorre 36-48h após a admissão do paciente no hospital. Muito mais do que o tempo, são as condições fisiológicas que vão determinar se o paciente está apto para ser submetido a uma nova operação.

A correção definitiva das lesões deverá ser realizada nesse momento. Após a umidificação com solução salina aquecida, as compressas serão cuidadosamente retiradas para que não haja novos sangramentos. Os reparos vasculares necessários devem ser realizados, as coleções, retiradas, e, também, o desbridamento de tecidos necrosados deve ser feito.

O trânsito intestinal deve, nesse instante, ser restaurado com as anastomoses necessárias. Houve, por algum tempo, um certo consenso sobre a realização de anastomoses tardias após lesões extensas de cólon. Isso evitava a criação de estomas em pacientes cuja anastomose primária não era possível e, ao mesmo tempo, não aumentava o número de complicações ${ }^{39}$. A anastomose tardia dentro da reoperação programada era considerada factível, caso não houvesse acidose grave, edema de parede intestinal e/ou infecção intra-abdominal ${ }^{40}$. Entretanto, um estudo retrospectivo trouxe novamente à tona essa discussão. Weinberg et al. observaram um aumento significativo da incidência de complicações pós-operatórias nos pacientes submetidos à ressecção e anastomose tardia em relação aos pacientes submetidos à ressecção e colostomia terminal tardia ( $12 \%$ vs. $3 \%, p<0,05)^{41}$. Isso levanta, mais uma vez, a dúvida e nos leva a pensar que a realização de estomas pode ser a alternativa mais segura, apesar de ter suas próprias complicações.

\section{Fechamento da parede abdominal}

O fechamento definitivo do abdome pode muitas vezes ser um grande desafio para os cirurgiões, especialmente se o intervalo entre os dois procedimentos for maior que cinco dias. Durante esse período os músculos e suas fáscias contraem lateralmente deixando um grande defeito na linha mediana, o que impossibilita o fechamento convencional da parede abdominal. A sutura da aponeurose sob tensão não deve ser realizada, pois, além de ineficaz, pode predispor o paciente à síndrome compartimental abdominal ${ }^{3,5}$.

Se há uma dificuldade na síntese, algumas técnicas podem ser empregadas, como a utilização de telas (absorvíveis ou não absorvíveis). Deve-se evitar ao máximo o contato desses materiais (marlex, prolene) com as alças intestinais, pois o risco de formação de fístulas enterocutâneas pode chegar a $25 \%^{3}$.

Para evitar o aparecimento de grandes hérnias ventrais e o risco de fístulas, algumas novas técnicas vêm sendo utilizadas, como o curativo com aspiração contínua por vácuo e o dispositivo Wittmann Patch ${ }^{\circledR}$, citados por Kushimoto et al. ${ }^{3}$. Apesar de facilitarem a aproximação 
das bordas, esses são equipamentos caros e ainda pouco utilizados (Figuras 2 e 3$)^{42}$. Outra opção é a secção da bainha anterior dos músculos reto abdominais com a rotação de um retalho medialmente, protegendo, assim, as alças intestinais ${ }^{3}$ (Figura 4).

\section{Novas perspectivas}

O conceito de cirurgia abreviada vem se estendendo fora do cenário de trauma abdominal. Uma revisão publicada em 2006, discute as possibilidades do emprego do controle de danos em traumas torácicos. Essa abordagem segue o mesmo princípio de corrigir de maneira temporária as lesões e também busca técnicas mais simples e rápidas para repará-las ${ }^{6}$. Um relato de série de casos demonstrou que a toracotomia abreviada em pacientes gravemente traumatizados pode reduzir a mortalidade ${ }^{43}$.

Já se discute se a cirurgia para controle de danos pode melhorar a sobrevida de pacientes cirúrgicos gravemente enfermos com instabilidade hemodinâmica decorrente de sepse grave, de hemorragia volumosa, de isquemia mesentérica aguda ou de infecções necrotizantes. Esses possíveis fatores em conjunto com a necessidade de irrigações peritoneais repetidas, a fim de erradicar focos infecciosos, e a necessidade de reavaliar suturas e anastomoses podem ser eventuais indicações para a cirurgia de controle de danos em casos de emergência não decorrente de trau$\mathrm{ma}^{4}$.

Um estudo retrospectivo baseado nos scores APACHE II e POSSUM, com 16 pacientes, demonstrou uma queda na mortalidade de pacientes com hemorragia intraoperatória, isquemia mesentérica, sepse e pancreatite necrotizante que foram submetidos a laparotomia abrevia$\mathrm{da}^{7}$.

\section{Considerações Finais}

A aplicação da cirurgia de controle de danos já é rotina no manejo do abdome de pacientes gravemente traumatizados. Apesar de não existirem estudos multicêntricos prospectivos randomizados controlados, essa abordagem é aceita e reconhecida por cirurgiões especialistas na área. A experiência clínica, até o presente momento, já demonstrou que quando feita de maneira correta e, mais importante, quando bem indicada, reduz a mortalidade em pacientes com traumas ${ }^{1,5}$. Por ser uma área dentro da cirurgia relativamente nova e pouco explorada, é muito provável que novas técnicas e abordagens apareçam nos próximos anos e aumentem a sobrevida desses pacientes.

\section{Agradecimentos}

À Gabriel Berta, pela confecção das ilustrações inseridas nesse trabalho.

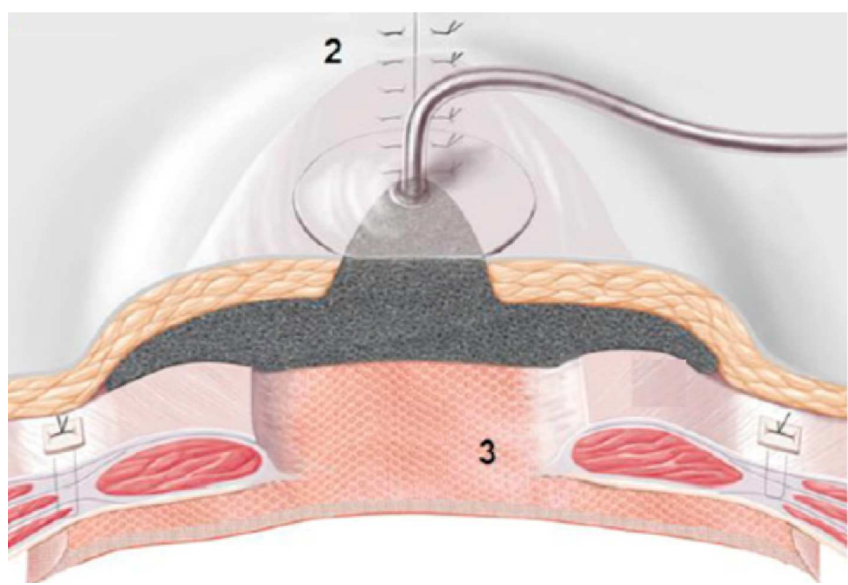

Figura 3 - $\quad$ Representação esquemática do curativo a vácuo com tela para fechamento da parede abdominal. (2. Curativo a vácuo com esponja de poliuretano colocada entre a fáscia e o tecido subcutâneo. 3. Tela sintética com formação de tecido de granulação) - Imagem original em Dietz UA et al. 201242.
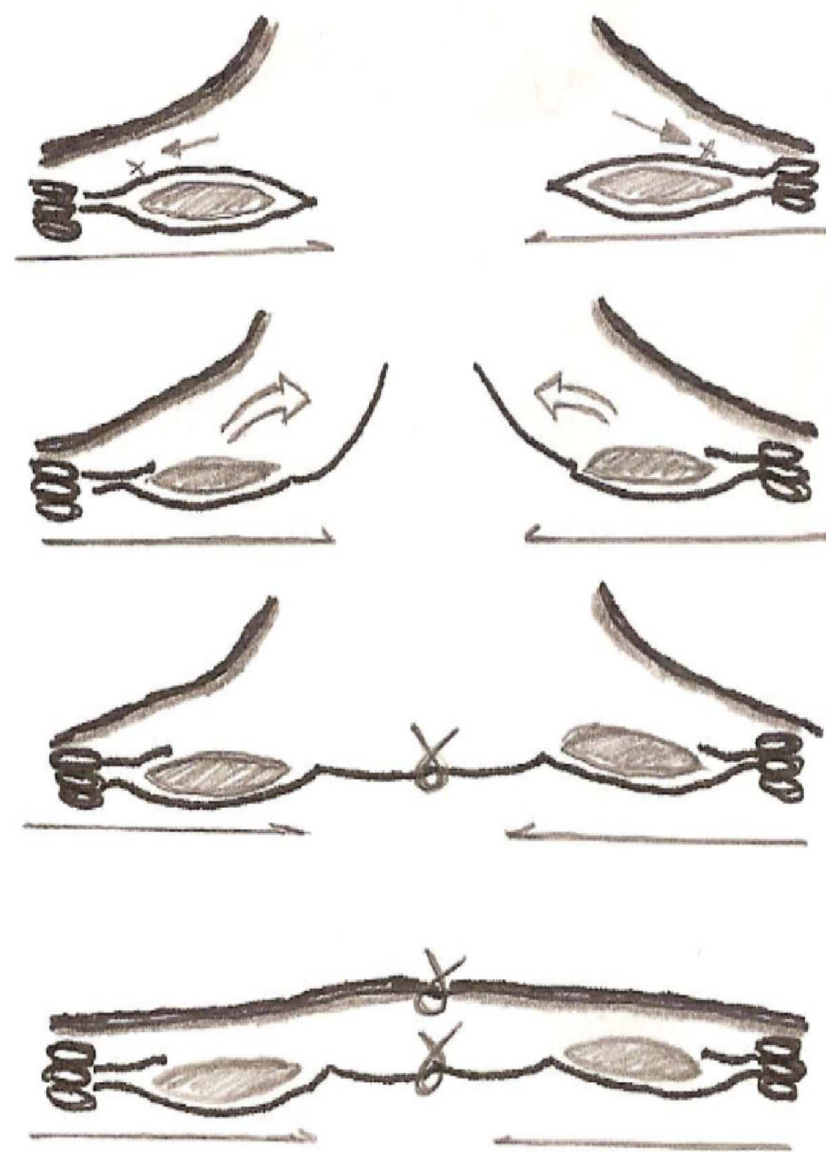

Figura 4 - $\quad$ Fechamento da parede abdominal com a rotação medial da bainha anterior dos músculos reto abdominal (Modificado de Kushimoto et al. ${ }^{3}$ ). 
The damage control surgery is a widely accepted concept today among abdominal trauma specialists when it comes to the severely traumatized. In these patients, the death is due, in most cases, to the installation of the lethal triad (hypothermia, coagulopathy and acidosis) and not the inability to repair the serious initial damage. In this review, the authors address the lethal triad in its three phases and emphasize the measures taken to prevent them, as well as discussing the indication and employment of damage control surgery in its various stages. Restoring the physiological status of the patient in the ICU, so that he/she can be submitted to final operation and closure of the abdominal cavity, another challenge in severe trauma patients, is also discussed.

Key words: Patients. Wounds and injuries. Therapy. Operative surgical procedures. Control.

\section{REFERÊNCIAS}

1. Germanos S, Gourgiotis S, Villias C, Bertucci M, Dimopoulos N, Salemis N. Damage control surgery in the abdomen: an approach for the management of severe injured patients. Int J Surg. 2008;6(3):246-52.

2. Jaunoo SS, Harji DP. Damage control surgery. Int J Surg. 2009;7(2):110-3.

3. Kushimoto S, Miyauchi M, Yokota H, Kawai M. Damage control surgery and open abdominal management: recent advances and our approach. J Nippon Med Sch. 2009;76(6):280-90.

4. Jansen JO, Loudon MA. Damage control surgery in a non-trauma setting. Br J Surg. 2007;94(7):789-90

5. Parreira JG, Soldá S, Rasslan S. Controle de danos: uma opção tática no tratamento dos traumatizados com hemorragia grave. Arq gastroenterol. 2002;39(3):188-97

6. Phelan HA, Patterson SG, Hassan MO, Gonzalez RP, Rodning CB. Thoracic damage-control operation: principles, techniques, and definitive repair. J Am Coll Surg. 2006;203(6):933-41.

7. Stawicki SP, Brooks A, Bilski T, Scaff D, Gupta R, Schwab CW, et al. The concept of damage control: extending the paradigm to emergency general surgery. Injury. 2008;39(1):93-101.

8. Stone HH, Strom PR, Mullins RJ. Management of the major coagulopathy with onset during laparotomy. Ann Surg. 1983;197(5):532-5.

9. Rotondo MF, Schwab CW, McGonigal MD, Phillips GR 3rd, Fruchterman TM, Kauder DR, et al. 'Damage control': an approach for improved survival in exsanguinating penetrating abdominal injury. J Trauma. 1993;35(3):375-82; discussion 382-3.

10. Burch JM, Ortiz VB, Richardson RJ, Martin RR, Mattox KL, Jordan GL Jr. Abbreviated laparotomy and planned reoperation for critically injured patients. Ann Surg. 1992;215(5):476-83; discussion 483-4.

11. Murthi SB, Stansbury LG, Dutton RP, Edelman BB, Scalea TM, Hess JR. Transfusion medicine in trauma patients: an update. Expert Rev Hematol. 2011;4(5):527-37.

12. Kairinos N, Hayes PM, Nicol AJ, Kahn D. Avoiding futile damage control laparotomy. Injury. 2010;41(1):64-8.

13. Holcomb JB. Damage control resuscitation. J Trauma. 2007;62(6Suppl):S36-7.

14. Moore EE, Burch JM, Franciose RJ, Offner PJ, BiffI WL. Staged physiologic restoration and damage control surgery. World I Surg. 1998;22(12):1184-90; discussion 1190-1.

15. Matsumoto $H$, Mashiko K, Sakamoto $Y$, Kutsukata N, Hara $Y$, Yokota $\mathrm{H}$. A new look at criteria for damage control surgery. I Nippon Med Sch. 2010;77(1):13-20

16. Rotondo MF, Zonies $\mathrm{DH}$. The damage control sequence and underlying logic. Surg Clin North Am. 1997;77(4):761-77.

17. Lima RAC, Rocco PRM. Cirurgia para controle do dano. Rev Col Bras Cir. 2007;34(4):257-63.

18. Asensio JA, McDuffie L, Petrone P, Roldán G, Forno W, Gambaro $E$, et al. Reliable variables in the exsanguinated patient which indicate damage control and predict outcome. Am J Surg. 2001;182(6):743-51.
19. Parreira JG, Soldá SC, Rasslan S. Análise dos indicadores de hemorragia letal em vítimas de trauma penetrante de tronco admitidas em choque: um método objetivo para selecionar os candidatos ao controle de danos. Rev Col Bras Cir. 2002;29(5):256-66.

20. Higa G, Friese R, O'Keeffe T, Wynne J, Bowlby P, Ziemba M, et al. Damage control laparotomy: a vital tool once overused. J Trauma. 2010;69(1):53-9

21. Cosgriff N, Moore EE, Sauaia A, Kenny-Moynihan M, Burch JM, Galloway B. Predicting life-threatening coagulopathy in the massively transfused trauma patient: hypothermia and acidoses revisited. J Trauma. 1997;42(5):857-61; discussion 861-2.

22. Cushman JG, Feliciano DV, Renz BM, Ingram WL, Ansley JD, Clark WS, et al. Iliac vessel injury: operative physiology related to outcome. J Trauma. 1997;42(6):1033-40.

23. Garrison JR, Richardson JD, Hilakos AS, Spain DA, Wilson MA, Miller FB, et al. Predicting the need to pack early for severe intraabdominal hemorrhage. J Trauma. 1996;40(6):923-7; discussion 927-9.

24. Krishna G, Sleigh JW, Rahman H. Physiological predictors of death in exsanguinating trauma patients undergoing conventional trauma surgery. Aust N Z J Surg. 1998;68(12):826-9.

25. Stalhschmidt CMM, Formighieri B, Lubachevski FL. Controle de danos no trauma abdominal e lesões associadas: experiência de cinco anos em um serviço de emergência. Rev Col Bras Cir. 2002;33(4):215-9.

26. Fávero SS, Corsi PR, Coimbra RS, Rasslan S. Treatment of transfixing hepatic lesions with a hidrostatic balloon. Sao Paulo Med J. 1994:112(4):629-34.

27. Feliciano D, Moore EE, Mattox KL. Trauma. $3^{\text {a }}$ ed. Stanford: Appleton Lange; 1996. Damage control and alternative wound closures in abdominal trauma; p.717-31.

28. Feliciano D, Moore EE, Mattox KL. Trauma. $4^{\text {a }}$ ed. New York: McGraw-Hill; 2000. Damage control and alternative wound closures in abdominal trauma; p.907-32.

29. Pachter HL, Spencer FC, Hofstetter SR, Liang HG, Coppa GF. Significant trends in the treatment of hepatic trauma. Experience with 411 injuries. Ann Surg. 1992;215(5):492-500; discussion 5002 .

30. CRASH-2 collaborators, Roberts I, Shakur H, Afolabi A, Brohi K, Coats $\mathrm{T}$, et al. The importance of early treatment with tranexamic acid in bleeding trauma patients: an exploratory analysis of the CRASH-2 randomised controlled trial. Lancet. 2011:377(9771):1096-101.e1-2.

31. Borgman MA, Spinella PC, Perkins JG, Grathwohl KW, Repine T, Beekley AC, et al. The ratio of blood products transfused affects mortality in patients receiving massive transfusions at a combat support hospital. J Trauma. 2007;63(4):805-13.

32. Jansen JO, Thomas R, Loudon MA, Brooks A. Damage control resuscitation for patients with major trauma. BMJ. 2009;338:b1778.

33. Fabron Júnior A, Lopes LB, Bordin JO. Lesão pulmonar aguda associada à transfusão. J bras pneumol. 2007;33(2):206-12.

34. Hannon T. Trauma blood management: avoiding the collateral damage of trauma resuscitation protocols. Hematology Am Soc Hematol Educ Program. 2010;2010:463-4. 
35. Waibel BH, Rotondo MF. Damage control surgery: it's evolution over the last 20 years. Rev Col Bras Cir. 2012;39(4):314-21.

36. Asensio JA, Demetriades D, Chahwan S, Gomez H, Hanpeter D, Velmahos $G$, et al. Approach to the complex hepatic injuries. J Trauma. 2000:48(1):66-9.

37. NICE-SUGAR Study Investigators, Finfer S, Chittock DR, Su SY, Blair D, Foster D, et al. Intensive versus conventional glucose control in critically ill patients. N Engl J Med. 2009;360(13):1283-97.

38. Ventilation with lower tidal volumes as compared with traditional tidal volumes for acute lung injury and the acute respiratory distress syndrome. The Acute Respiratory Distress Syndrome Network. N Engl J Med. 2000;342(18):1301-8.

39. Miller PR, Chang MC, Hoth JJ, Holmes JH 4th, Meredith JW. Colonic resection in the setting of damage control laparotomy: is delayed anastomosis safe? Am Surg. 2007;73(6):606-9; discussion 609-10.

40. Ordoñez CA, Pino LF, Badiel M, Sánchez Al, Loaiza J, Ballestas L, et al. Safety of performing a delayed anastomosis during damage control laparotomy in patients with destructive colon injuries. J Trauma. 2011;71(6):1512-7; discussion 1517-8.

41. Weinberg JA, Griffin RL, Vandromme MJ, Melton SM, George RL, Reiff DA, et al. Management of colon wounds in the setting of damage control laparotomy: a cautionary tale. J Trauma. 2009;67(5):929-35.
42. Dietz UA, Wichelmann C, Wunder C, kauczok J, Spor L, Strauâ A, et al. Early repair of open abdomen with a tailored two-component mesh and conditioning vacuum packing: a safe alternative to the planned giant ventral hernia. Hernia. 2012;16(4):451-60.

43. Vargo DJ, Battistella FD. Abbreviated thoracotomy and temporary chest closure: an application of damage control after thoracic trauma. Arch Surg. 2001;136(1):21-4.

Recebido em 08/03/2012

Aceito para publicação em 15/08/2012

Conflito de interesse: nenhum

Fonte de financiamento: nenhuma

\section{Como citar este artigo:}

Edelmuth RCL, Buscariolli YS, Ribeiro Júnior MAF. Cirurgia para controle de danos: estado atual. Rev Col Bras Cir. [periódico na Internet] 2013;40(2). Disponível em URL: http://www.scielo.br/rcbc

\section{Endereço para correspondência:}

Rodrigo Camargo Leão Edelmuth

E-mail: rodrigo.edelmuth@me.com 